« Cooperative provision of indivisible public goods »

\author{
$\underline{\text { Auteurs }}$
}

Pierre Dehez

Document de Travail n 2010 - 14

Juin 2010

Faculté des sciences économiques et de gestion Pôle européen de gestion et d'économie (PEGE) 61 avenue de la Forêt Noire F-67085 Strasbourg Cedex

Secétariat du BETA Géraldine Manderscheidt Tél. : (33) 0368852069 Fax : (33) 0368852070 g.manderscheidt@unistra.fr http://cournot2.u-strasbg.fr/beta 


\title{
Cooperative provision of indivisible public goods
}

\author{
Pierre Dehez \\ CORE (University of Louvain, Louvain-la-Neuve) \\ BETA (CNRS - Universities of Strasbourg and Nancy) \\ pierre.dehez@uclouvain.be
}

May 2010

\begin{abstract}
A community faces the obligation of providing an indivisible public good. Each member is capable of providing it at a certain cost and the solution is to rely on the player who can do it at the lowest cost. It is then natural that he or she be compensated by the other players. The question is to know how much they should each contribute. We model this compensation problem as a cost sharing game to which standard allocation rules are applied and related to the solution resulting from the auction procedures proposed by Kleindorfer and Sertel (1994).
\end{abstract}

JEL: C71, H41, M41

Keywords: public goods, cost sharing, core, nucleolus, Shapley value 


\section{Introduction}

A community faces the obligation of providing a public good (or service) for its own benefit. It is assumed that the public good is indivisible and that each agent is capable of providing it at a certain cost. The quality of the public good does not depend on who provides it and individual costs are publicly known. The question is not to know whether the public good should be provided, nor in which quantity. The question is to know who should provide the public good and how much each agent should contribute. The division of labor within a household or the location of noxious facilities within a region are possible illustrations of such a problem.

This problem was first studied by Kunreuther et al. (1987) in a mechanism design perspective and later by Kleindorfer and Sertel (1994) who proposed an auction procedure whereby each agent makes a bid specifying the compensation he or she would ask for in exchange of being the provider. The public good is then provided by the agent having made the lowest bid and the required compensation is equally divided among the non-providers. This defines a noncooperative game whose Nash equilibria induce a solution satisfying the following properties:

- efficiency the public good is provided by the lowest cost agent

- fairness the non-providers contribute an identical amount

- non envy the non-providers have no interest in providing the public good, the provider has no interest in non-providing the public good

These three properties define an interval of individual contributions, henceforth called "KS" solution.

To be more precise, let $c_{i}$ denote the cost associated to agent $i(i=1, \ldots, n)$ and assume that $c_{1}$ and $c_{2}$ are the lowest and next lowest cost i.e. $0 \leq c_{1}<c_{2}$ and $c_{2} \leq c_{i}$ for all $i \neq 1,2$. Efficiency requires that the public good should be provided by agent 1 . Fairness and non-envy lead to an identical contribution $t \geq 0$ from each non-provider satisfying the following inequalities:

$$
\begin{aligned}
& c_{1}-(n-1) t \leq t \\
& c_{i}-(n-1) t \geq t \text { for all } i \neq 1
\end{aligned}
$$

or

$$
\frac{1}{n} c_{1} \leq t \leq \frac{1}{n} c_{i} \text { for all } i \neq 1
$$

Hence, individual contributions must be within the interval $T(c)=\left[c_{1} / n, c_{2} / n\right]$. The lower bound is the equal division: every agent supports the same share in the lowest cost. Indeed, the provider's net cost is then given by $c_{1}-(n-1) c_{1} / n=c_{1} / n$. What about the nature of the upper bound? We observe that the provider's net contribution may fall below the 
contributions of the non-providers, depending upon the extend of the provider's competitive advantage measured by the cost difference $c_{2}-c_{1}$. It may even be negative: providing the public good may be a source of revenue. A second question concerns the choice of a particular compensation level within the interval $T(c)$.

We approach these questions from a cooperative point of view using games with transferable utility. Given a cost vector $c=\left(c_{1}, \ldots, c_{n}\right)$, we define a cost sharing game henceforth called "compensation game". The core of a compensation game has interesting properties. It is nonempty and includes the allocations associated to KS contributions. Like the KS solution, it depends exclusively on the two lowest cost components. Furthermore, it has a regular structure. More precisely, the core is a regular simplex. As a consequence, the nucleolus coincides with its center of gravity that, in this case, is the average of core's vertices. It define a particular KS solution, namely its upperbound $c_{2} / n$.

A compensation game is a kind of "reverse" airport game and its Shapley value is defined by a simple formula equivalent to the solution of the airport game. While core allocations only compensate the lowest cost player, the Shapley value often compensate other players as well, rendering it inadequate as a compensation rule in this context. ${ }^{1}$ The Shapley value is indeed based on players' marginal costs and therefore it takes the entire cost distribution into account. We show that the Shapley value belongs to the core if and only if no more than one player is compensated.

The analysis is easily extended to the provision of several public goods. The resulting compensation game is the sum of the compensation games associated to individual public goods. We show that the core and the nucleolus are additive on the class of compensation games.

The paper is organized as follows. The auction procedure proposed by Kleindorfer and Sertel is formalized in Section 2. Cost games and airport games are introduced in Section 3. The compensation game associated to the provision of an indivisible public good is defined in Section 4. It is shown to be monotonically decreasing and subadditive. The core and the nucleolus of a compensation game are characterized in the two subsequent sections. Section 7 is devoted to the Shapley value. The extension to the simultaneous provision of several indivisible public goods is treated in Section 8. The concluding section includes a discussion on the nature of the cost components.

\footnotetext{
${ }^{1}$ See Dehez and Tellone (2010) for a compensation problem where the Shapley value is a compensation rule that appears more appropriate than the nucleolus.
} 


\section{Auctioning an indivisible public good}

Each player has the capacity to provide the public good at a given cost, $c_{i}$ for player $i$. Without loss of generality, we order players in terms of their cost:

$$
0 \leq c_{1} \leq c_{2} \leq \ldots \leq c_{n}
$$

with $c_{n}>0$.

Kleindorfer and Sertel (1994) have proposed an auction procedure whereby each player submits a sealed bid stipulating the compensation he or she requires to provide the public good. For a given a bid profile $b=\left(b_{1}, \ldots, b_{n}\right) \in \mathbb{R}^{n}$, the provider is identified as the first lowest bidder:

$$
i(b)=\operatorname{Min}\left\{j \mid b_{j}=\ell(b)\right\}
$$

where the lowest bid $\ell(b)=\operatorname{Min}_{i \in N} b_{i}$ is equally divided among the other players. ${ }^{2}$ The utility for player $i$ associated to a bid profile $b=\left(b_{1}, \ldots, b_{n}\right)$ is then given by:

$$
u_{i}\left(b_{1}, \ldots, b_{n}\right)=\begin{array}{ll}
\ell(b)-c_{i} & \text { if } i=i(b) \\
\frac{-1}{n-1} \ell(b) & \text { if } i \neq i(b)
\end{array}
$$

This defines a game in normal form. Kleindorfer and Sertel show that any Nash equilibrium $\bar{b}=\left(\bar{b}_{1}, \ldots, \bar{b}_{n}\right)$ of this game identifies player 1 as the provider, $i(\bar{b})=1$, who receives a compensation $\ell(\bar{b})=\bar{b}_{1}$ satisfying

$$
\rho_{1}(c) \leq \ell(\bar{b}) \leq \rho_{2}(c) \text { where } \rho_{i}(c)=\frac{n-1}{n} c_{i}
$$

where the interval $\left[\rho_{1}(c), \rho_{2}(c)\right]$ corresponds to the interval of individual contributions $T(c)=\left[c_{1} / n, c_{2} / n\right]$ defined in the introduction. Moreover, any compensation in the interval $\left[\rho_{1}(c), \rho_{2}(c)\right]$ can be associated to a Nash equilibrium i.e. compensations in $\left[\rho_{1}(c), \rho_{2}(c)\right]$ are fully Nash implementable through that simple auction procedure. ${ }^{3}$ Hence $\rho_{2}(c)$ is the maximum equilibrium bid by player 1 . Incidentally, $\rho_{i}(c)$ is the maximin (prudent) bid by player $i$.

In the case where $c_{1}=c_{2}$ there is a unique Nash equilibrium since the interval then reduces to a single point: $T(c)=\left\{c_{1} / n\right\}$.

\footnotetext{
${ }^{2}$ Ties are broken by refering to the natural order of the players.

${ }^{3}$ Actually Kleindorfer and Sertel show that compensation in $R(c)$ are Nash implementable by an auction procedure of the " $k^{\text {th }}$ lowest bidder" type $(2 \leq k \leq n)$ with full implementation if the number of players having a cost equal to the minimum cost does not exceed $n-k+1$.
} 


\section{Cost games and airport games}

A set $N=\{1, \ldots, n\}$ of players, $n \geq 2$, have a common project and face the problem of dividing its cost. The cost of realizing the project to the benefit of the members of any given coalition is also known. This defines a real-valued function $C-$ a cost function - on the subsets of $N$. By convention, it satisfies $C(\varnothing)=0$. The pair $(N, C)$ is a cost game. ${ }^{4}$ A sharing rule $\varphi$ associates a cost allocation $y=\varphi(N, C)$ to any cost game $(N, C)$ such that

$$
\sum_{i=1}^{n} \varphi_{i}(N, C)=C(N)
$$

Airport games form a particular class of cost games. ${ }^{5}$ The project is to build a facility (e.g. a runway) capable of meeting all players' needs. The cost of a facility meeting player $i$ 's needs is denoted by $c_{i}, c_{i} \geq 0$. It is assumed that the facility whose $\operatorname{cost}$ is $c_{i}$ also covers the needs of players with a lower cost. The associated cost game $(N, C)$ is then defined by:

$$
C(S)=\operatorname{Max}_{i \in S} c_{i}
$$

It is a monotonically increasing and concave (and thereby subadditive) game:

$$
\begin{aligned}
& S \subset T \Rightarrow C(S) \leq C(T) \\
& S, T \subset N \Rightarrow C(S)+C(T) \geq C(S \cup T)+C(S \cap T)
\end{aligned}
$$

Notation: The letters $n, s, t, \ldots$ denote the size of the sets $N, S, T, \ldots$ For a vector $x, x(S)$ denotes the sum over $S$ of its coordinates, with $x(\varnothing)=0$ by convention. Coalitions are identified as $i j k \ldots$ instead of $\{i, j, k\} \ldots$ For any set $S, S \backslash i$ denotes the coalition from which player $i$ has been removed.

\section{Compensation games}

Consider a cost vector $c=\left(c_{1}, \ldots, c_{n}\right)$ satisfying (1). If a coalition forms, it relies on the player with the lowest cost. The associated compensation game $(N, C)$ is then defined by:

$$
C(S)=\operatorname{Min}_{i \in S} c_{i} \text { for all } S \subset N, S \neq \varnothing
$$

with $C(\varnothing)=0$. In particular $C(N)=c_{1}$. The cost function $C$ can alternatively be written as:

$$
C(S)=-C_{0}(S) \text { where } C_{0}(S)=\operatorname{Max}_{i \in S}\left(-c_{i}\right)
$$

However $C_{0}$ does not define a proper airport game, because its cost components are negative. In particular, the function $C_{0}$ is superadditive.

\footnotetext{
${ }^{4}$ For an introduction to the theory of cost allocation, see Young (1985).

${ }^{5}$ Airport games were introduced by Littlechild and Owen (1973). See Thomson (2007) for a complete survey.
} 
Lemma 1 Compensation games are monotonically decreasing, subadditive and essential.

Proof Consider a compensation game $(N, C)$ as defined by (2). For any two coalitions $S$ and $T$ in $N$, we have successively:

$$
\begin{aligned}
& S \subset T \Rightarrow C(T)-C(S)=\operatorname{Min}_{i \in T} c_{i}-\operatorname{Min}_{i \in S} c_{i} \leq 0 \\
& S \cap T=\varnothing \Rightarrow C(S)+C(T)=\operatorname{Min}_{i \in T} c_{i}+\operatorname{Min}_{i \in S} c_{i} \geq \operatorname{Min}_{i \in S \cup T} c_{i}=C(S \bigcup T)
\end{aligned}
$$

Furthermore,

$$
c_{n}>0 \Rightarrow \sum_{i \in N} C(i)=\sum_{i \in N} c_{i}>\operatorname{Min}_{i \in N} c_{i}=C(N)
$$

Hence the game $(N, C)$ is monotonically decreasing, subadditive and essential.

Compensation games are generally not concave except in special cases like for instance if the $n-1$ last cost components are equal.

Lemma 2 The compensation game defined by an ordered cost vector $c \in \mathbb{R}_{+}^{n}$ such that $0 \leq c_{1} \leq c_{2}=\ldots=c_{n}$ is concave.

Proof Let us define $\Delta=C(S)+C(T)-C(S \cup T)-C(S \cap T)$. To check for concavity, we have to verify that $\Delta \geq 0$ for all $S$ and $T$ in $N$.

The case where $S \cap T=\varnothing$ is covered by subadditivity (actually $\Delta=c_{2}$ once $S \cap T=\varnothing$ ).

If $S$ and $T$ are such that $S \cap T \neq \varnothing$, there are four possible cases:

$$
\begin{aligned}
& 1 \in S \backslash T \text { or } 1 \in T \backslash S \\
& 1 \in S \cap T \\
& 1 \notin S \cup T
\end{aligned}
$$

It is easily verified that $\Delta=0$ in all three cases.

Example 1 The 3-player game associated to the cost vector $c=(3,9,15)$ is defined by:

$$
\begin{aligned}
& C(1)=C(12)=C(13)=C(123)=3 \\
& C(2)=C(23)=9 \\
& C(3)=15
\end{aligned}
$$

Here $c_{1}=15$ and the cost to be divided is 3 . 
Example 2 The 4-player game associated to the cost vector $c=(0,3,9,12)$ is defined by:

$$
\begin{aligned}
& C(S)=0 \text { for all coalition } S \text { containing player } 1 \\
& C(2)=C(23)=C(24)=C(234)=3 \\
& C(3)=C(34)=9 \\
& C(4)=12
\end{aligned}
$$

Here $c_{1}=0$ and the cost to be divided is 0 .

\section{The core}

An imputation $y$ is an individually rational cost allocation:

$$
y(N)=C(N) \text { and } y(i) \leq C(i) \text { for all } i \in N
$$

The set of imputations is denoted by $I(N, C)$. For essential and subadditive cost games, $I(N, C)$ is a non-empty subset of $\mathbb{R}^{n}$ of dimension $n-1$. The core is the set of imputations against which no coalition can object:

$$
\mathbb{C}(N, C)=\left\{y \in \mathbb{R}^{n} \mid y(N)=c_{1} \text { and } y(S) \leq C(S) \text { for all } S \subset N\right\}
$$

i.e. no coalition pays more that its stand alone cost. ${ }^{6}$ Equivalently, an imputation $y$ belongs to the core if and only if there is no cross-subsidization ${ }^{7}$ in the sense that every coalition pays at least its marginal cost:

$$
\mathbb{C}(N, C)=\left\{y \in \mathbb{R}^{n} \mid y(N)=c_{1} \text { and } y(S) \geq C(N)-C(N \backslash S) \text { for all } S \subset N\right\}
$$

In general, the core is a convex polyhedron, possibly empty. Its maximal dimension is $n-1$.

The core of a compensation game is non-empty. It contains the allocations corresponding to the KS contributions:

$$
K S(c)=\left\{y \in \mathbb{R}^{n} \mid y=\left(c_{1}-(n-1) t, t, \ldots, t\right), t \in T(c)\right\}
$$

Indeed if $y \in K S(c)$ then $y(N)=c_{1}$ and

$$
y(S)=\begin{aligned}
& s t \leq s \frac{c_{2}}{n} \leq c_{2} \leq C(S) \quad \text { if } 1 \notin \mathrm{S} \\
& c_{1}-\frac{s-1}{n-1} t \leq c_{1}=C(S) \quad \text { if } 1 \in \mathrm{S}
\end{aligned}
$$

\footnotetext{
${ }^{6}$ The concept of core of a TU-game was introduced by Gillies (1953).

${ }^{7}$ See Faulhaber (1975).
} 
The core also contains the no-compensation allocation $y^{0}=\left(c_{1}, 0, \ldots, 0\right)$. Indeed, we have:

$$
y^{0}(N)=c_{1} \text { and } y^{0}(S) \leq c_{1} \leq C(S) \text { for all } S \subset N
$$

The following proposition establishes that the core of a compensation game is a regular simplex. ${ }^{8}$ It has full dimension if $c_{2}>0$ and reduces to $\{0\}$ if $c_{1}=c_{2}=0$. Furthermore, the core depends only on the two lowest cost components $c_{1}$ and $c_{2}$.

Proposition 1 The core of the compensation game defined by the ordered cost vector $c \in \mathbb{R}_{+}^{n}$ is a regular simplex whose $n$ vertices are:

$$
\begin{aligned}
& v^{1}=\left(c_{1}, 0, \ldots, 0\right) \\
& v^{2}=\left(c_{1}-c_{2}, c_{2}, \ldots, 0\right) \\
& v^{3}=\left(c_{1}-c_{2}, 0, c_{2}, 0, \ldots, 0\right) \\
& \ldots \\
& v^{n}=\left(c_{1}-c_{2}, 0, \ldots 0, c_{2}\right)
\end{aligned}
$$

It has full dimension if $c_{2}>0$ and $\mathbb{C}(N, C)=\{0\}$ if $c_{1}=c_{2}=0$.

Proof Using the alternative definition of the core given by (4), the core of a compensation game can be written simply as

$$
\mathbb{C}(N, c)=\left\{y \in \mathbb{R}^{n} \mid y(N)=c_{1}, \quad y_{1} \geq c_{1}-c_{2} \text { and } y_{i} \geq 0 \text { for all } i \neq 1\right\}
$$

Indeed, if $y \in \mathbb{C}(N, c)$ we have successively:

$$
\begin{aligned}
& y(N \backslash i) \leq C(N \backslash i)=c_{1} \text { for all } i \neq 1 \\
& y(N \backslash 1) \leq C(N \backslash 1)=c_{2}
\end{aligned}
$$

(6) then follows from $y(N)=c_{1}$. If now $y$ satisfies (6) and $S \subset N$, we have successively:

$$
\begin{aligned}
& \text { if } 1 \in S: y(N \backslash S) \geq 0 \quad \Rightarrow y(S) \leq c_{1}=C(S) \\
& \text { if } 1 \notin S: y(N \backslash S) \geq c_{1}-c_{2} \Rightarrow y(S) \leq c_{2} \leq C(S)
\end{aligned}
$$

Hence, $y \in \mathbb{C}(N, c)$. Translating the core - as defined in (6) - by adding the vector $\left(c_{2}-c_{1}, 0, \ldots, 0\right)$, we obtain the standard simplex $\left\{y \in \mathbb{R}_{+}^{n} \mid y(N)=c_{2}\right\} .{ }^{9}$ It has full dimension if $c_{2}>0$ and $\mathbb{C}(N, c)=\{0\}$ if $c_{1}=c_{2}=0$.

\footnotetext{
${ }^{8}$ A simplex in $\mathbb{R}^{n}$ is the convex hull of $n$ affinely independent vectors. A simplex is a polyhedral set. A facet is a maximal proper face of a polyhedral set. See Grünbaum (2003).

${ }^{9}$ If $c_{2}>0$, the unit simplex $\Delta_{n}=\left\{y \in \mathbb{R}_{+}^{n} \mid y(N)=1\right\}$ is obtained by dividing by $c_{2}$.
} 
Hence the core of a compensation game is an equilateral triangle for $n=3$, a regular tetrahedron for $n=4, \ldots$ The distance between vertices is equal to $c_{2} \sqrt{2}$. Its $n$ facets have dimension $n-2$. They are given by:

$$
\begin{aligned}
& F_{1}=\left\{y \in \mathbb{R}^{n} \mid y(N)=c_{1}, \quad y_{1}=c_{1}-c_{2}\right\}=\left\{y \in \mathbb{R}^{n} \mid y(N)=c_{1}, y(N \backslash 1)=c_{2}\right\} \\
& F_{i}=\left\{y \in \mathbb{R}^{n} \mid y(N)=c_{1}, \quad y_{i}=0\right\}=\left\{y \in \mathbb{R}^{n} \mid y(N)=c_{1}, y(N \backslash i)=c_{1}\right\} \quad(i=2, \ldots n)
\end{aligned}
$$

The following figure illustrates Proposition 1 for the case where $n=3$ and $0<c_{1}<c_{2}$.

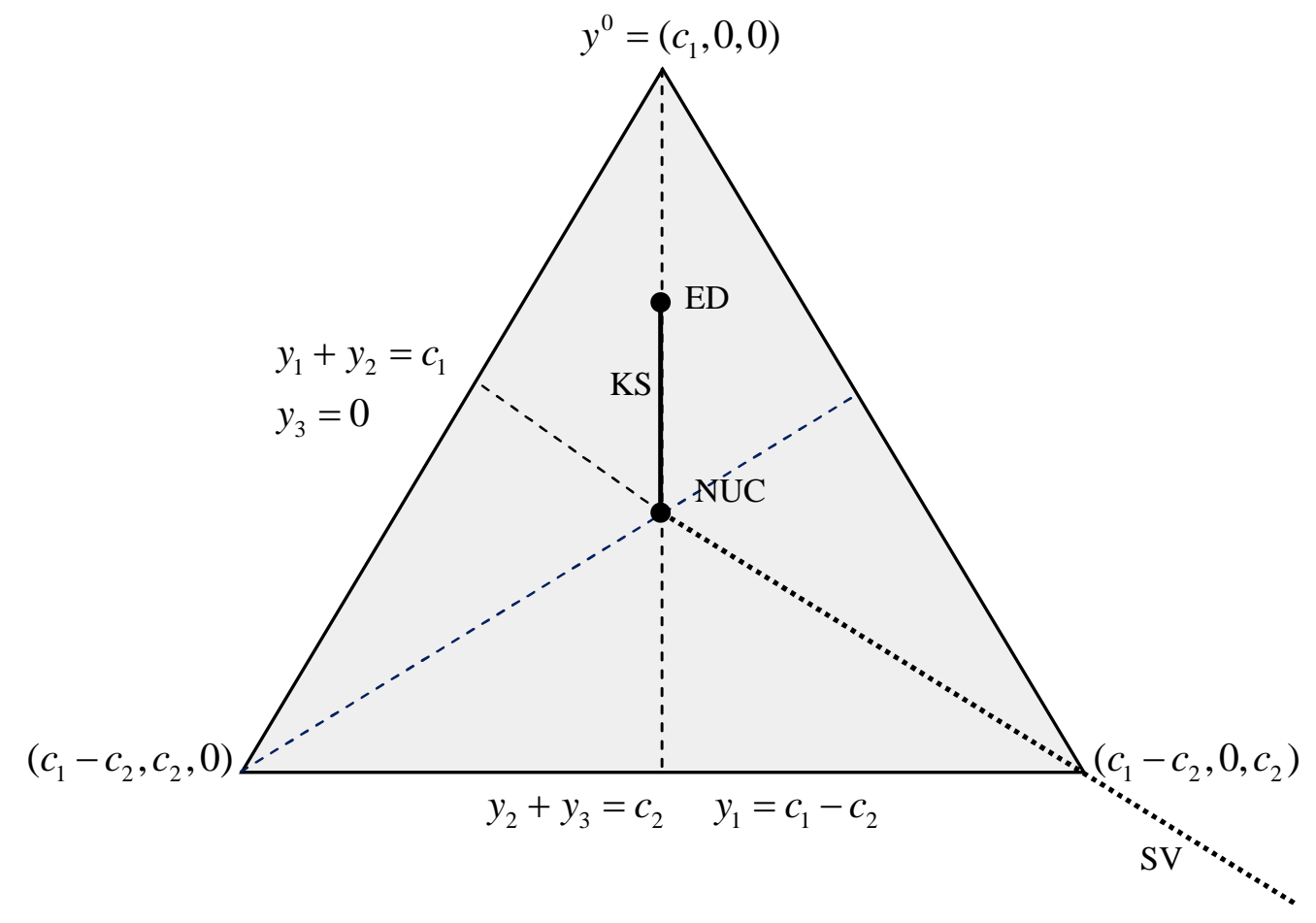

Referring to (6), we observe that the equal division (ED) allocation $\bar{y}=\left(c_{1} / n, \ldots, c_{1} / n\right)$ also belongs to the core. Except in the case where $c_{1}=c_{2}, \bar{y}$ differs from the centre of gravity of the core that, by regularity, is here defined by the average of core's vertices: ${ }^{10}$

$$
\left(c_{1}-\frac{n-1}{n} c_{2}, \frac{c_{2}}{n}, \ldots, \frac{c_{2}}{n}\right)
$$

We observe that the core of the compensation game $(N, C)$ defined by the ordered cost vector $c \in \mathbb{R}_{+}^{n}$ coincides with the core of the concave cost game $(N, \bar{C})$ defined by the ordered cost vector $\bar{c} \in \mathbb{R}_{+}^{n}$ where $\overline{c_{1}}=c_{1}$ and $\bar{c}_{i}=c_{2}$ for all $i \neq 1$. The marginal cost vectors associated to the game $(N, \bar{C})$ are then precisely the core's vertices (5).

\footnotetext{
${ }^{10}$ The centre of gravity of the core has been introduced as a solution concept by Gonzales-Diaz and SanchezRodriguez (2007).
} 


\section{The nucleolus}

For an imputation $y \in I(N, C)$ and a coalition $S \subset N(S \neq \varnothing, N)$, we define the gain of coalition $S$ at the imputation $y$ :

$$
e(y, S)=C(S)-y(S)
$$

as the difference between the cost of coalition $S$ and what it contributes under $y$. The least core and the nucleolus (NUC) are solution concepts that are concerned with the maximization of these gains. An imputation $y$ belongs to the core if $e(y, S) \geq 0$ for all $S \subset N$. The least core is the set of imputations that maximizes the lowest loss:

$$
\operatorname{Max}_{y \in I(N, C)} \operatorname{Min}_{\substack{S \subset N \\ S \neq \varnothing, N}} e(y, S)
$$

It has dimension at most $n-2$. If the core is non-empty, the least core is obviously a subset of the core. The nucleolus introduced by Schmeidler (1969) goes further by comparing excesses lexicographically so as to eventually retain a unique allocation.

We have $C(N \backslash 1)=c_{2}$ and $C(N \backslash i)=c_{1}$ for all $i \neq 1$. Using (7), the facets of the core of a compensation game can be written as

$$
F_{i}=\left\{y \in \mathbb{R}^{n} \mid y(N)=c_{1}, \quad y(N \backslash i)=C(N \backslash i)\right\} \quad i=1, \ldots n
$$

where

$$
\begin{aligned}
& C(N \backslash 1)=c_{2} \\
& C(N \backslash i)=c_{1} \text { for all } i \neq 1
\end{aligned}
$$

The definition of the least core then simplifies to:

$$
\operatorname{Max}_{y \in I(N, C)} \operatorname{Min}_{i \in N} e(y, N \backslash i)
$$

By regularity of the core, the least core is the set of allocations $y$ such that $y(N)=c_{1}$ and

$$
e(y, N \backslash i)=a \text { for all } i \in N
$$

for some real $a$. Using (8), we get:

$$
\begin{aligned}
& e(y, N \backslash 1)=y(N \backslash 1)-c_{2}=\left(c_{1}-y_{1}\right)-c_{2} \\
& e(y, N \backslash i)=y(N \backslash i)-c_{1}=\left(c_{1}-y_{i}\right)-c_{1}=-y_{i} \quad(i=2, \ldots, n)
\end{aligned}
$$

The solution is given by:

$$
\begin{aligned}
& y_{1}=c_{1}-c_{2}-a \\
& y_{i}=-a
\end{aligned} \quad \text { where } a=-\frac{c_{2}}{n} .
$$


The least core being uniquely defined, it coincides with the nucleolus. This proves the following proposition.

Proposition 2 The nucleolus of the compensation game $(N, C)$ defined by the ordered cost vector $c \in \mathbb{R}_{+}^{n}$ depends only on the two lowest cost components $c_{1}$ and $c_{2}$. It is given by:

$$
\eta(N, c)=\left(c_{1}-\frac{n-1}{n} c_{2}, \frac{c_{2}}{n}, \ldots, \frac{c_{2}}{n}\right)=\left(\frac{c_{2}}{n}-\left(c_{2}-c_{1}\right), \frac{c_{2}}{n}, \ldots, \frac{c_{2}}{n}\right)
$$

The lowest cost player supports the cost of producing the public good and receives a compensation from the other players. It is proportional to the second lowest cost and all contribute the same amount. Applying (9) to the the examples 1 and 2, we obtain the allocations $(-3,3,3)$ and $(-2.25,0.75,0.75,0.75)$ respectively.

Given core's regularity, the nucleolus coincides with the centre of gravity of the core: it is located at equal distance from the facets and at equal distance from the vertices. Computing the nucleolus is simple and only requires the identification of the two lowest cost components.

As illustrated in the figure, the equal division allocation $\bar{y}$ is located on the segment joining the nucleolus to the no-compensation allocation $v^{1}=y^{0}$ depending on the value of $c_{1}$ : $\bar{y}=v^{1}=0$ if $c_{1}=0$ and $\bar{y}=\eta(N, c)$ if $c_{1}=c_{2}$.

Equal charge is a well known accounting rule. It is defined by:

$$
\theta_{i}(N, C)=C(N)-C(N \backslash i)+\frac{1}{n}\left(C(N)-\sum_{j=1}^{n}(C(N)-C(N \backslash j))\right) \quad i=1, \ldots, n
$$

Applying it to the compensation game defined by the ordered cost vector $c \in \mathbb{R}_{+}^{n}$ and using (8), we obtain exactly (9) i.e. $\eta(N, c)=\theta(N, c)$.

\section{The Shapley value}

Consider a cost game $(N, C)$ and the set of player's permutations $\Pi_{N}$. To each permutation $\pi=\left(i_{1}, \ldots, i_{n}\right) \in \Pi_{N}$ we associate a marginal cost vector that is the allocation $t(\pi)$ whose elements are given by:

$$
\begin{aligned}
& t_{i_{1}}(\pi)=C\left(i_{1}\right) \\
& t_{i_{k}}(\pi)=C\left(i_{1}, \ldots, i_{k}\right)-C\left(i_{1}, \ldots, i_{k-1}\right) \text { for } k=2, \ldots, n
\end{aligned}
$$

The Shapley value is then simply the average marginal cost vector

$$
\varphi_{i}(N, C)=\frac{1}{n !} \sum_{\pi \in \Pi_{N}} t_{i}(\pi)
$$


It is the unique additive sharing rule that satisfies symmetry and dummy. ${ }^{11}$ Alternative axioms have been proposed. ${ }^{12}$ The Shapley value is individually rational for subadditive cost games. It does not necessarily belong to the core except for some classes of games like concave cost games.

Proposition 3 The Shapley value of the compensation game defined by the ordered cost vector $c \in \mathbb{R}_{+}^{n}$ is given by the allocation $\varphi(N, c)$ where:

$$
\begin{aligned}
& \varphi_{n}(N, c)=\frac{c_{n}}{n} \\
& \varphi_{n-1}(N, c)=\frac{c_{n}}{n}+\frac{c_{n-1}-c_{n}}{n-1} \\
& \ldots \\
& \varphi_{1}(N, c)=\frac{c_{n}}{n}+\frac{c_{n-1}-c_{n}}{n-1}+\ldots+\frac{c_{2}-c_{3}}{2}+c_{1}-c_{2}
\end{aligned}
$$

Proof The allocation defined by (10) is actually the Shapley value of the airport game defined by a cost vector $\left(c_{1}, \ldots, c_{n}\right)$ satisfying the inequalities $c_{n} \leq c_{n-1} \leq \ldots \leq c_{1} \cdot{ }^{13}$

Using the linearity of the Shapley value and the alternative definition (3) of a compensation game $(N, C)$ we get:

$$
\varphi(N, C)=-\varphi\left(N, C_{0}\right)
$$

Because the cost vector satisfies reversed inequalities $-c_{n} \leq-c_{n-1} \leq \ldots \leq-c_{1}$, we then obtain exactly (10).

We observe that the Shapley value and the nucleolus coincide in the particular case where $c_{i}=c_{2}$ for all $i \geq 3$. It is a consequence of the convavity of the game and the regularity of the core.

The recursive structure of the formula allows the Shapley value to be written in matrix form as $y=A$. $c$ where $A$ is a $n \times n$ triangular matrix whose elements are:

$$
a_{i j}=\frac{-1}{j(j-1)} \text { for all } j>i \text { and } a_{i i}=\frac{1}{i} \text { for all } i
$$

with $a_{i j}=0$ otherwise.

\footnotetext{
${ }^{11}$ These are the original axioms used by Shapley (1953): players with identical marginal costs pay the same amount (symmetry) and players with zero marginal costs pay nothing (dummy). The nucleolus satisfies symmetry and dummy but not additivity.

${ }^{12}$ See for instance Young (1985). In the context of cost sharing it is shown that the Shapley sharing rule is the unique rule which allocates fixed costs uniformly. See Dehez (2009).

${ }^{13}$ See Littlechild and Owen (1973).
} 
Applying (10) to the examples 1 and 2, we obtain the allocations $(-4,2,5)$ and $(-4,-1,2,3)$ respectively. Only the first allocation belongs to the core because in the second, two players are compensated.

Proposition 4 The allocation $y$ derived from the Shapley value of the compensation game defined by the ordered cost vector $c \in \mathbb{R}_{+}^{n}$ belongs to the core if and only if none of the last $n-1$ players are compensated: $y_{i} \geq 0$ for all $i \neq 1$.

Proof Let $y=\varphi(N, C)$ be the allocation derived from the Shapley value (10). If $y$ belongs to the core, we know from (6) that $y_{i} \geq 0$ for all $i \neq 1$. To prove the "if" part, we observe that $y_{2} \geq 0$ implies $y_{1}=y_{2}+\left(c_{1}-c_{2}\right) \geq c_{1}-c_{2}$. Hence (6) is verified if $y_{i} \geq 0$ for all $i \neq 1$.

Actually, it suffices to check that $y_{2} \geq 0$. Indeed, the allocation $y$ derived from the Shapley value satisfies $y_{n} \geq y_{n-1} \geq \ldots y_{1}$. Hence $y_{2} \geq 0$ implies $y_{i} \geq 0$ for all $i \neq 1$. This gives a single the condition on the $c_{i}$ 's under which the Shapley value belongs to the core:

$$
\frac{c_{2}}{2} \geq \sum_{j=3}^{n} \frac{1}{j(j-1)} c_{j}
$$

We observe that this inequality is independent of the lowest cost $c_{1}$. For $n=3$, it reduces to $c_{3} \leq 3 c_{2}$. Graphically, the Shapley value is located along the line segment (SV) starting at the nucleolus for $c_{3}=c_{2}$ and passing through the lower-right vertex $\left(c_{1}-c_{2}, 0, c_{2}\right)$ for $c_{3}=3 c_{2}$. For $n=4$, the inequality reads $c_{4}+2 c_{3} \leq 6 c_{2}$.

\section{Extension to several public goods}

Let $M=\{1, \ldots, m\}$ be the set of public goods to be supplied. A cost vector is associated to each public good, $a^{h} \in \mathbb{R}_{+}^{n}$ for good $h \in M$. The resulting compensation game is denoted by $\left(N, C_{h}\right)$. The cost game $(N, C)$ associated to the simultaneous provision of the $m$ public goods is the sum of the individual compensation games:

$$
C(S)=\sum_{h \in M} C_{h}(S) \text { where } C_{h}(S)=\operatorname{Min}_{i \in S} a_{i}^{h}
$$

This is a general compensation game. Its core has a simple relationship to the core of the elementary compensation games $\left(N, C_{h}\right)$.

Proposition 5 The core of a general compensation game is the sum of the cores of the elementary compensation games:

$$
\mathbb{C}(N, C)=\sum_{h \in M} \mathbb{C}\left(N, C_{h}\right)
$$

It is a regular simplex whose vertices are the sum of the vertices of the cores of the elementary games. 
Proof We denote by $c_{1}^{h}$ and $c_{2}^{h}$ the two lowest costs of producing public good $h$, with $0 \leq c_{1}^{h} \leq c_{2}^{h}$. We shall first show that the core of the general compensation game can be written as:

$$
\mathbb{C}(N, C)=\left\{y \in \mathbb{R}^{n} \mid y(N)=\sum_{h \in M} c_{1}^{h}, y_{i} \geq \sum_{h \in M_{i}}\left(c_{1}^{h}-c_{2}^{h}\right) \text { for all } i\right\}
$$

where $M_{i}=\left\{h \in M \mid c_{i}^{h}=c_{1}^{h}<c_{2}^{h}\right\}$ is the set of public goods for which player $i$ has the strictly lowest cost (recalling that sums over empty sets are zero.) The sets $M_{0}, M_{1}, \ldots, M_{n}$ form a partition of $M$ where $M_{0}=\left\{h \in M \mid c_{1}^{h}=c_{2}^{h}\right\}$.

(i) If $y \in \mathbb{C}(N, C)$ we have:

$$
y(N \backslash j) \leq \sum_{h \in M} C(N \backslash j)=\sum_{h \in M_{j}} c_{2}^{h}+\sum_{h \in M \backslash M_{j}} c_{1}^{h} \text { for all } j \in N
$$

Since $y(N)=\sum_{h \in M} c_{1}^{h}$ we get:

$$
y_{j} \geq \sum_{h \in M_{j}} c_{1}^{h}-\sum_{h \in M_{j}} c_{2}^{h} \text { for all } j \in N
$$

(ii) If $y$ satisfies (11) and $S \subset N$, we have successively:

and

$$
C(S)=\sum_{h \in M} C_{h}(S) \geq \sum_{i \in S} \sum_{h \in M_{i}} c_{1}^{h}+\sum_{i \in N \backslash S} \sum_{h \in M_{i}} c_{2}^{h}+\sum_{h \in M_{0}} c_{1}^{h}
$$

$$
\begin{aligned}
& y(N \backslash S) \geq \sum_{i \in N \backslash S} \sum_{h \in M_{i}}\left(c_{1}^{h}-c_{2}^{h}\right) \\
& \Rightarrow y(S) \leq \sum_{h \in M} c_{1}^{h}-\sum_{i \in N \backslash S} \sum_{h \in M_{i}}\left(c_{1}^{h}-c_{2}^{h}\right)=\sum_{h \in M_{0}} c_{1}^{h}+\sum_{i \in S} \sum_{h \in M_{i}} c_{1}^{h}+\sum_{i \in N \backslash S} \sum_{h \in M_{i}} c_{2}^{h}
\end{aligned}
$$

Hence $y(S) \leq C(S)$ for all $S \subset N$ and $y \in \mathbb{C}(N, C)$.

(iii) Translating (11) by adding the vector $b \in \mathbb{R}^{n}$ defined by

$$
b_{i}=\sum_{h \in M_{i}}\left(c_{2}^{h}-c_{1}^{h}\right) \quad i=1, \ldots, n
$$

we obtain the standard simplex $\left\{y \in \mathbb{R}_{+}^{n} \mid y(N)=\sum_{h \in M} c_{2}^{h}\right\}$. Indeed

$$
\begin{aligned}
& y \in \mathbb{C}(N, C) \text { and } z=y+b \\
& \Rightarrow \quad z_{i} \geq 0 \text { and } z(N)=\sum_{h \in M_{0}} c_{1}^{h}+\sum_{i \in N} \sum_{h \in M_{i}} c_{2}^{h}=\sum_{h \in M} c_{2}^{h}
\end{aligned}
$$

recalling that $c_{1}^{h}=c_{2}^{h}$ for all $h \in M_{0}$. This confirms that $\mathbb{C}(N, C)$ is a simplex. 
(iv) The $n$ vertices $v^{1}, \ldots, v^{n}$ of $\mathbb{C}(N, C)$ are obtained by subtracting $b$ from the vertices of the simplex $\left\{y \in \mathbb{R}_{+}^{n} \mid y(N)=\sum_{h \in M} c_{2}^{h}\right\}$. Following (5), they are defined by:

$$
\begin{aligned}
v_{i}^{j} & =\sum_{h \in M_{i}} c_{1}^{h}+\sum_{h \in M \backslash M_{i}} c_{2}^{h}=\sum_{h \in M} v_{i}^{j}(h) & & \text { if } i=j \\
& =\sum_{h \in M_{i}}\left(c_{1}^{h}-c_{2}^{h}\right)=\sum_{h \in M} v_{i}^{j}(h) & & \text { if } i \neq j
\end{aligned}
$$

where $v^{1}(h), \ldots, v^{n}(h)$ are the vertices of the elementary game $\left(N, C_{h}\right)$ given by:

$$
\begin{aligned}
& \text { for all } h \in M \backslash M_{0} \text { and } i=i^{h} \quad v_{i}^{j}(h)=c_{1}^{h} \quad \text { if } j=i \\
& =c_{1}^{h}-c_{2}^{h} \quad \text { if } j \neq i \\
& \text { for all } h \in M \backslash M_{0} \text { and } i \neq i^{h} \quad v_{i}^{j}(h)=c_{2}^{h} \quad \text { if } j=i \\
& =0 \quad \text { if } j \neq i \\
& \text { for all } h \in M_{0} \text { and for all } i \quad v_{i}^{j}(h)=c_{2}^{h} \quad \text { if } j=i \\
& =0 \quad \text { if } j \neq i
\end{aligned}
$$

where for all $h \in M \backslash M_{0}, i^{h}$ denotes the lowest cost player i.e. $a_{i^{h}}=c_{1}^{h}$. This concludes the proof of Proposition 1.

The core of a general compensation game being a regular simplex, the nucleolus coincides with the center of gravity that is easily characterized using (12). Furthermore, it is the sum of the nucleoli associated to the elementary compensation games.

Corollary The nucleolus of the aggregate compensation game is given by:

$$
\eta_{i}(N, C)=\frac{1}{n} \sum_{h \in M} c_{2}(h)-\sum_{h \in M_{i}}\left(c_{2}(h)-c_{1}(h)\right)=\sum_{h \in M} \eta_{i}\left(N, C_{h}\right)
$$

Hence, the core and the nucleolus (as well as the Shapley value) are additive on the set of all general compensation games.

Example 3 Consider the combination of Example 1 with the game associated to the cost vector $a^{2}=(18,12,15)$. Given the cost vector $a^{1}=(3,9,15)$ used in Example 1 , the resulting compensation game is given by:

$$
\begin{aligned}
& C(1)=21, C(2)=21, C(3)=30 \\
& C(12)=15, C(13)=18, C(23)=21 \\
& C(123)=15
\end{aligned}
$$


Its nucleolus is the sum of the nucleoli: $(2,5,8)=(-3,3,3)+(5,2,5)$. The public goods are provided by player 1 and player 2 respectively.

\section{Concluding remarks}

The paper of Kleindorfer and Sertel (1994) provides non-cooperative foundations to the interval $T(c)$ of individual contributions resulting from the properties of efficiency, fairness and non-envy. The present paper provides cooperative foundations. In both approaches, the question of the nature of the cost components emerges naturally. The answer depends on the context.

If the problem concerns a chore within a household, like for instance moving the garbage, some may be willing to pay more than others to avoid that unpleasant task. This is reflected in the individual cost components that measure the disutility associated to that task. The individual cost components may as well reflect the capacity - physical and/or mental - of each player to carry a particular task within a community.

If the problem concerns the location of a noxious facility, like for instance a waste incinerator, $c_{i}$ is understood as the nuisance value for player $i$ expressed in monetary terms, to which one may add the cost of building the facility. This is however not necessary if this cost is independent of who host the facility. This is a fixed cost that should be divided equally among the players. Indeed, if $\tilde{c}$ is the cost vector defined by $\tilde{c}_{i}=c_{i}+F$ for some fixed $\operatorname{cost} F>0$,

$$
t \in T(c) \text { if and only if } t+\frac{F}{n} \in T(\tilde{c})
$$

and the nucleolus is given by:

$$
\eta_{i}(N, \tilde{c})=\eta_{i}(N, c)+\frac{F}{n} \text { for all } i=1, \ldots, n
$$

The cost component may also include a possible benefit related to being provider. This is the case if the problem concerns the location of a desirable facility. ${ }^{14}$ Our analysis covers such cases if the benefit never exceeds the cost, ensuring that the $c_{i}$ are all non-negative. This excludes the case where some players would be willing to pay to be provider. The auction procedure proposed by Kleindorfer and Sertel still applies in this case but the stability of KS contributions is not ensured: some (or all) associated cost allocations may not belong to the core. KS contributions may even fail to be individually rational. The problem is that the compensation game is not subadditive, resulting in an empty core. There may even be no

\footnotetext{
${ }^{14}$ Kleindorfer and Sertel suggest the location of European institutions or scientific meetings as possible examples.
} 
imputations. This is for instance the case for the cost vector $c=(-6,-3,2)$ for which we have:

$$
T(c)=[-2,-1] \text { and } K S(c)=\left\{y \in \mathbb{R}^{3} \mid y=(-6-2 t, t, t),-2 \leq t \leq-1\right\}
$$

Obviously no KS allocation is individually rational. This indicates that the non-negativity of the cost components is an essential assumption. This also suggests that the way the problem is modeled here is not appropriate to a situation where some players are willing to pay to be provider while some others are willing to be compensated.

\section{References}

Dehez P. (2009), The allocation of fixed costs and the weighted Shapley value, CORE Discussion Paper 2009/35.

Dehez, P. and D. Tellone (2010), Data games. Sharing the cost of public goods with exclusion, Revised CORE Discussion Paper 2008-10.

Faulhaber G. (1975), Cross-subsidization: Pricing in public enterprises, American Economic Review 65, 966-977.

Gillies D.B. (1953), Some theorems on $n$-person games. PhD Thesis, University of Princeton.

Gonzáles-Díaz J. and E. Sánchez-Rodríguez (2007), A natural selection from the core of a TU game: the core-centre, International Journal of Game Theory 36, 27-46.

Grünbaum B. (2003), Convex polytopes, $2^{\text {nd }}$ edition, Berlin: Springer Verlag.

Kleindorfer P. and M. Sertel (1994), Auctioning the provision of an indivisible public good, Journal of Economic Theory 64, 20-34.

Kunreuther H., P. Kleindorfer, P.J. Knetz and R. Yaksick (1987), A compensation mechanism for siting noxious facilities: Theory and experimental design, Journal of Environmental Economics and Management 14, 371-383.

Littlechild S. C. and G. Owen (1973), A simple expression for the Shapley value in a special case, Management Science 20, 370-372.

Shapley L.S. (1953), A value for n-person games, in: Kuhn H. and A.W. Tucker, eds., Contributions to the Theory of Games II, Princeton: Princeton University Press, 307-317.

Schmeidler, D. (1969), The nucleolus of a characteristic function game, SIAM Journal of Applied Mathematics 17, 1163-1170.

Thomson W. (2007), Airport problems and cost allocation, Rochester Center for Economic Research Working Paper.

Young, P.Y. (1985), Cost Allocation: Methods, Principles, Application, Amsterdam: NorthHolland. 


\section{Documents de travail du BETA}

2010-01 The Aggregation of Individual Distributive Preferences through the Distributive Liberal Social Contract : Normative Analysis Jean MERCIER-YTHIER, janvier 2010.

2010-02 Monnaie et Crise Bancaire dans une Petite Economie Ouverte Jin CHENG, janvier 2010.

2010-03 A Structural nonparametric reappraisal of the $\mathrm{CO}_{2}$ emissions-income relationships Theophile AZOMAHOU, Micheline GOEDHUYS, Phu NGUYEN-VAN, janvier 2010.

2010-04 The signaling role of policy action Romain BAERISWYL, Camille CORNAND, février 2010.

2010-05 Pro-development growth and international income mobility: evidence world-wide Jalal EL OUARDIGHI, mars 2010.

2010-06 The determinants of scientific research agenda: Why do academic inventors choose to perform patentable versus non-patentable research?

Caroline HUSSLER, Julien PENIN, mars 2010.

2010-07 Adverse Selection, Emission Permits and Optimal Price Differentiation Mourad AFIF, Sandrine SPAETER, mars 2010.

2010-08 The impact of ambiguity on health prevention and insurance Johanna ETNER, Sandrine SPAETER, mars 2010.

2010-09 Equité du plaider coupable : une analyse économétrique dans trois tribunaux de grande instance français.

Lydie ANCELOT, mars 2010.

2010-10 Networks, Irreversibility and Knowledge Creation.

Patrick LLERENA, Muge OZMAN, mars 2010.

2010-11 Les clusters et les réseaux comme fondements de la dynamique d'innovation dans l'industrie biopharmaceutique Marc Hubert DEPRET, Abelillah HAMDOUCH, avril 2010.

2010-12 Large-scale risks and technological change: What about limited liability? Julien JACOB, Sandrine SPAETER, avril 2010.

2010-13 Innovation and Development. The Evidence from Innovation Surveys Francesco BOGLIACINO, Giulio PERANI, Mario PIANTA, Stefano SUPINO, avril 2010.

2010-14 Cooperative provision of indivisible public goods

Pierre DEHEZ, juin 2010.

La présente liste ne comprend que les Documents de Travail publiés à partir du $1^{\mathrm{er}}$ janvier 2010 . La liste complète peut être donnée sur demande.

This list contains the Working Paper writen after January 2010, 1rst. The complet list is available upon request. 